\title{
How well do we teach the primary healthcare approach? A case study of health sciences course documents, educators and students at the University of Cape Town Faculty of Health Sciences
}

J Irlam, ${ }^{1}$ BSc, BSc Hons (Med), MSc, MPhil; M I Datay, ${ }^{1}$ MB ChB, DPhil, FCP; $\mathbf{S}$ Reid, ${ }^{1}$ BSc (Med), MB ChB, MFamMed, PhD; M Alperstein, ${ }^{2}$ BSocSci Nursing, PG Dip, MPhil; N Hartman, ${ }^{2}$ MSocSci, PhD; M Namane, ${ }^{3}$ MB ChB, MSc (Immunol), MPhil (Fam Med), MSc (Clin Epidemiol) S Singh, ${ }^{4}$ BSpHT, MA, PhD; F Walters, ${ }^{4}$ BSpHT, MSc

\author{
${ }^{1}$ Primary Health Care Directorate, Faculty of Health Sciences, University of Cape Town, South Africa \\ ${ }^{2}$ Department of Health Sciences Education, Faculty of Health Sciences, University of Cape Town, South Africa \\ ${ }^{3}$ Division of Family Medicine, Faculty of Health Sciences, University of Cape Town, South Africa \\ ${ }^{4}$ Division of Communication Sciences and Disorders, Faculty of Health Sciences, University of Cape Town, South Africa
}

Corresponding author: J Irlam (james.irlam@uct.ac.za)

\begin{abstract}
Background. The comprehensive primary healthcare (PHC) approach has been a lead theme in the University of Cape Town Faculty of Health Sciences (FHS) since 1994. A 2014 institutional academic review recommended that indicators be developed for monitoring and evaluating the PHC theme. Objective. To evaluate PHC teaching and learning of final-year health and rehabilitation sciences and medical students at three community-based education (CBE) sites of the faculty, two in Cape Town and one in a distant and largely rural district.

Methods. Course documents were analysed for evidence and alignment of nine indicators of the PHC approach in the documented learning outcomes, activities and assessments of final-year health sciences students. Clinical educators and students were interviewed to identify factors that facilitate or impede PHC teaching and learning on site.

Results. Final-year health sciences disciplines engage inconsistently with PHC principles at the CBE sites. Alignment appears to be strongest between learning outcomes and teaching activities, but the available data are insufficient to judge whether there is also strong alignment between outcomes and teaching, and formal graded assessment. PHC teaching and learning at the CBE sites are facilitated by good multiprofessional teamwork, educator rolemodelling and good infrastructural and logistical support. Language barriers, staff shortages and high workloads are significant and prevalent barriers. Conclusion. Strong faculty leadership is required to promote the PHC lead theme and to achieve better departmental and multiprofessional collaboration in teaching the PHC approach. This study provides evidence from well-established CBE sites to inform future work and participatory action research in promoting the PHC approach in teaching and learning in the FHS.
\end{abstract}

Afr J Health Professions Educ 2021;13(1):83-92. https://doi.org/10.7196/AJHPE.2021.v13i1.1284

In 1978, the landmark Declaration of Alma Ata on primary healthcare (PHC) recognised that a new approach was needed to achieve 'Health for All by the year 2000. ${ }^{[1]}$ In 1994, the University of Cape Town (UCT) Faculty of Health Sciences (FHS), recognising PHC-inspired health policy developments in a democratic South Africa (SA), adopted the PHC approach as a 'lead theme' in its teaching, research and clinical service. ${ }^{[2]}$ The multidisciplinary PHC Directorate was established in 2003 as a cross-cutting 'horizontal' unit to promote the PHC approach in the FHS, as well as expanding and managing the decentralised clinical teaching platform for more community-based education (CBE).

The concept of social accountability, or the capacity to respond to priority health needs and health system challenges, has gained currency in the past quarter century. ${ }^{[3,4]}$ It emphasises partnerships between health science faculties and key stakeholders in the health sector and communities, and the need for health sciences education to maximise its relevance and impact on people's health. ${ }^{[5,6]}$ The international Training for Health Equity Network (THEnet) was founded in 2008 to promote health equity through health workforce education, research and service, based on the principles of social accountability and community engagement. THEnet has developed a practical tool with extensive indicators to help health science faculties align their training of health workers with community needs: the Framework for Socially Accountable Health Workforce Education. ${ }^{[7]}$

An academic review of the PHC Directorate in October 2014 recommended, inter alia, that indicators be developed for monitoring and evaluating the PHC lead theme, which shares many principles with those of social accountability, such as equity, community partnerships and advocacy. All the authors of this article have extensive experience in teaching the PHC approach in the FHS, and most are members of the PHC Working Group, which was mandated by the FHS Deanery in June 2017 to respond to the recommendations of the academic review. The authors therefore formed an action research group to develop a set of indicators and evaluate PHC teaching and learning of final-year health and rehabilitation sciences and medical students at selected CBE sites of the FHS. The research group's objectives for the present study were therefore to identify evidence of selected PHC indicators in the documented learning outcomes, activities and assessments of final-year students, to evaluate the alignment of the 
outcomes, activities and assessments, and to identify factors that facilitate or impede teaching and learning of the $\mathrm{PHC}$ principles at the $\mathrm{CBE}$ sites. Congruent with the action research approach, a faculty-wide PHC symposium was planned from the outset to present the preliminary study findings to key stakeholders and collectively develop recommendations for promoting the PHC approach in the faculty.

\section{Methods}

A case study design was employed, examining three CBE sites that were deemed to have good potential for PHC teaching and learning by virtue of having permanent and dedicated site co-ordinator staff who were members of the PHC Directorate: the secondary-level New Somerset Hospital (NSH) in central Cape Town (which also has primary-level district functions); the primary-level Vanguard Community Health Centre (VCHC) in a nearby suburb; and Vredenburg District Hospital (VDH) in a largely rural district about $140 \mathrm{~km}$ north of Cape Town. The study received ethical clearance from the FHS Human Research Ethics Committee (ref. no. HREC 157/2018).

Case study designs facilitate the exploration of a phenomenon within its context using a variety of data sources, which are converged during data analysis to enhance credibility. ${ }^{[8]}$ The flexibility and rigour of the design makes it useful for developing theory, evaluating programmes and developing interventions in health science educational research. ${ }^{\left[{ }^{[9]}\right.}$

A national Delphi panel of experts in socially accountable health sciences education first selected a set of nine PHC indicators over two rounds (Table 1). Five indicators were adapted from the HEALTH FOR ALL mnemonic developed for students by the PHC Directorate (Fig. 1), and four from the Training for Health Equity Network Framework for Socially Accountable Health Workforce Education. ${ }^{[7]}$ The Delphi phase of the research is described in an article currently being prepared for publication by the team.

Course documents were then analysed for evidence of the Delphi phase indicators in final-year student learning outcomes, activities and assessments, by two members of the action research team. A family medicine specialist $(\mathrm{MN})$ analysed documents for medical courses, and a speech language therapist educator (FW) analysed documents from the health and rehabilitation sciences, as they are very familiar with the curriculum and have experience in curriculum design and review. Data collection sheets were designed to capture whether the indicators were present, absent or not relevant, as well as to record any examples or comments and the documentary source (course manuals, teaching and assessment tools, service-learning documents, the online course management system Vula). Each indicator had a descriptor to assist consistent understanding of what it represented.

All clinical educators with at least a year's experience at the CBE sites were invited by email to be interviewed individually by either JI or MID, who are experienced senior lecturers and PHC tutors in the PHC Directorate, and eight educators accepted. Use was made of a standard interview guide to elicit educators' understanding of the PHC approach, their perceptions of how evident the PHC principles were in student learning outcomes on site and what the main facilitators and barriers are to PHC teaching and learning (Table 2). A table of 18 PHC principles was used to check which principles are taught and/or assessed at each site.

All final-year students in the FHS with at least 3 weeks' exposure to one of the CBE sites were invited to focus group discussions via several Vula announcements. Eighteen students were eligible, nine responded initially, but only two speech language therapy (SLT) students and one medical student finally consented to participate. The SLT students were interviewed together, and the medical student alone. Two authors not involved in finalyear teaching or assessment (NH and MA) used an interview guide and the HEALTH FOR ALL mnemonic to elicit the students' understanding of PHC, their PHC learning experiences on site and their perspectives on facilitators and barriers (Table 2).

All interviews were audio-recorded with consent, transcribed and anonymised. Internal validity and credibility checks were done by summary and confirmation with interviewees during or after the interviews. The key themes were deduced from the transcripts during analysis.

\section{Results \\ Document analysis}

Course documents were requested from all heads of departments and conveners of final-year courses in the FHS. After three emailed requests, documents were received from family medicine, surgery, obstetrics and gynaecology $(\mathrm{O} \& \mathrm{G})$, paediatrics, physiotherapy (PT) and communication sciences and disorders (CSD, including audiology and SLT). Three disciplines responded that they do not teach on the CBE platform (orthopaedics, psychiatry and pharmacology), one agreed to participate but did not submit documents (rheumatology) and one did not respond (occupational therapy). The key findings are described in Tables $3 \mathrm{~A}$ and $\mathrm{B}$, which summarise the evidence of selected PHC indicators in the documents.

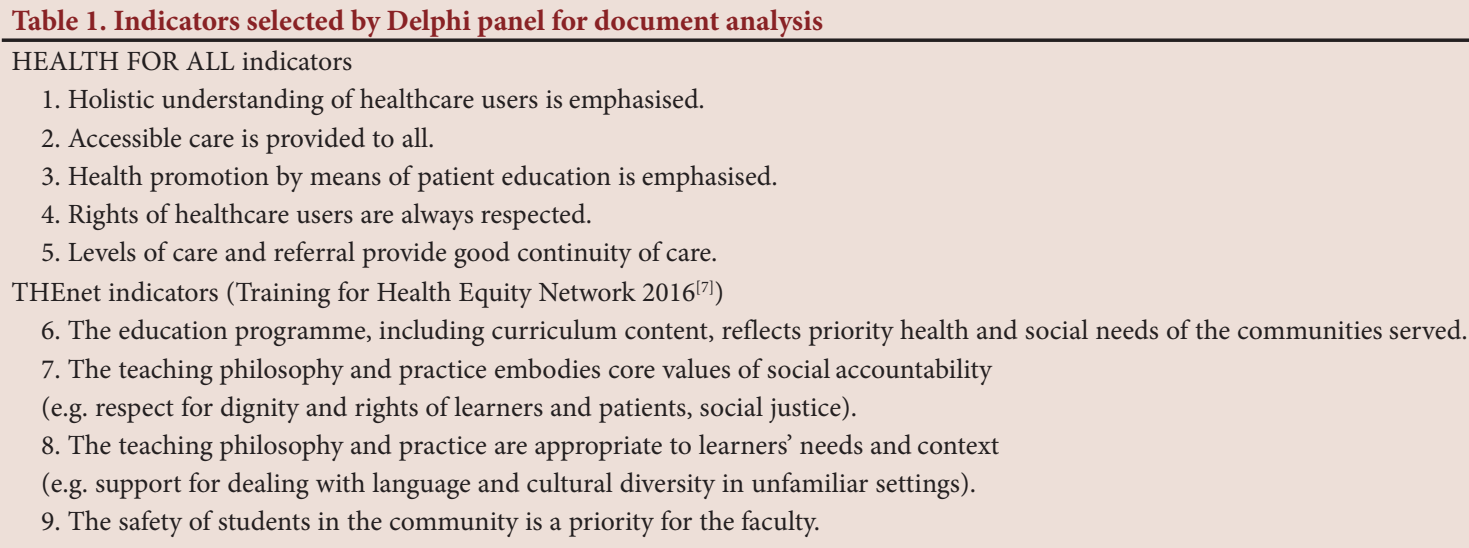

Table 1. Indicators selected by Delphi panel for document analysis

HEALTH FOR ALL indicators

1. Holistic understanding of healthcare users is emphasised.

2. Accessible care is provided to all.

3. Health promotion by means of patient education is emphasised.

4. Rights of healthcare users are always respected.

5. Levels of care and referral provide good continuity of care.

THEnet indicators (Training for Health Equity Network 2016 ${ }^{[7]}$ )

6. The education programme, including curriculum content, reflects priority health and social needs of the communities served.

7. The teaching philosophy and practice embodies core values of social accountability

(e.g. respect for dignity and rights of learners and patients, social justice).

8. The teaching philosophy and practice are appropriate to learners' needs and context

(e.g. support for dealing with language and cultural diversity in unfamiliar settings).

9. The safety of students in the community is a priority for the faculty. 


\begin{tabular}{|l|l|}
\hline Holistic & $\begin{array}{l}\text { Adopting a holistic, biopsychosocial ('whole person') } \\
\text { approach to health seekers, healthcare and health provision. }\end{array}$ \\
\hline Equity, Equality and Environment & $\begin{array}{l}\text { Being conscious of equity ('fairness') and equality requirements, } \\
\text { and emphasising ways to facilitate social justice in healthcare. } \\
\text { Recognising and responding to the impact of environmental concerns } \\
\text { (especially climate change) on health. }\end{array}$
\end{tabular}

Accessibility

Listening to, and learning from, communities

Teamwork (multidisciplinary)

\begin{tabular}{|l|}
\hline Health promotion \\
\hline Funding and resources (affordability)
\end{tabular}

\begin{tabular}{|l|l}
\hline Other sectors (intersectoral collaboration) \\
\hline
\end{tabular}

\begin{tabular}{|l|}
\hline Rights (human) and responsibilities \\
\hline
\end{tabular}

Acceptability and Appropriateness

Literature (evidence-based practice)

Levels of care and referral
Ensuring universal access to healthcare and provision.

Ensuring meaningful and reciprocal community and family liaison, engagement, participation and involvement in healthcare.

Commitment to multidisciplinary team approach to individual and public health.

Promoting individual and public health by means of information, education, communication, advocacy, mediation and enabling.

Committing appropriate resources (financial, structural, human, etc.) to ensure affordable quality healthcare.

Promoting broad intersectoral collaboration in addressing the social determinants of health.

Providing healthcare that is framed by and facilitates the realisation of human rights and responsibilities.

Ensuring that healthcare and provision is acceptable and appropriate for all users.

Providing healthcare that integrates critically appraised 'best' evidence from the literature with the patient's values and the clinician's experience.

Ensuring that levels of healthcare are appropriate and that referral processes are efficient.

Fig. 1. HEALTH FOR ALL mnemonic (Primary Health Care Directorate, University of Cape Town).

\section{Medical disciplines}

All four medical disciplines engage to varying extents with the nine PHC indicators. In paediatrics, all indicators and corresponding learning outcomes are explicitly stated in the 6th-year course manual, and the principle of human rights is apportioned a quarter of the ward mark. Family medicine promotes accessible care by teaching in rural and urban communities, and extending palliative care into patients' homes. Paediatrics is the only medical discipline with documentary evidence of the THEnet indicator of social accountability. Prioritising the safety of learners is evident in the 'Keep Safe' UCT student guide on Vula and in general safety awareness posters around campus. Specific advice about disease prevention and infection control is only evident in the paediatrics course manual.

\section{Health and rehabilitation disciplines}

All five HEALTH FOR ALL indicators were evident in the PT and CSD documents. Continuity of care is taught and assessed at all levels of the healthcare system, and CSD students do referrals with help from their UCT clinical supervisors, since there are no permanent Western Cape Department of Health staff to manage the caseload. The International Classification of Functioning, Disability and Health (ICF), which is the World Health Organization framework for measuring health and disability at individual and population levels, ${ }^{[10]}$ emphasises a holistic understanding of clients' needs. There were multiple examples of respect for human rights in references to informed consent, respect for privacy, professional communication and learning key words and phrases in the client's home language. PT students make services accessible in settings such as factories, childcare centres and patients' homes, and CSD students see both booked and unbooked patients. Health promotion is a central principle, so PT and CSD students undertake compulsory health promotion projects during their site rotations.

Most of the four THEnet indicators were evident. In addition to the 'Keep Safe' guide and tuberculosis policy on Vula, there is profession-specific advice about infection control, such as cleaning audiology equipment and 
Table 2. Interview questions for clinical educators and students

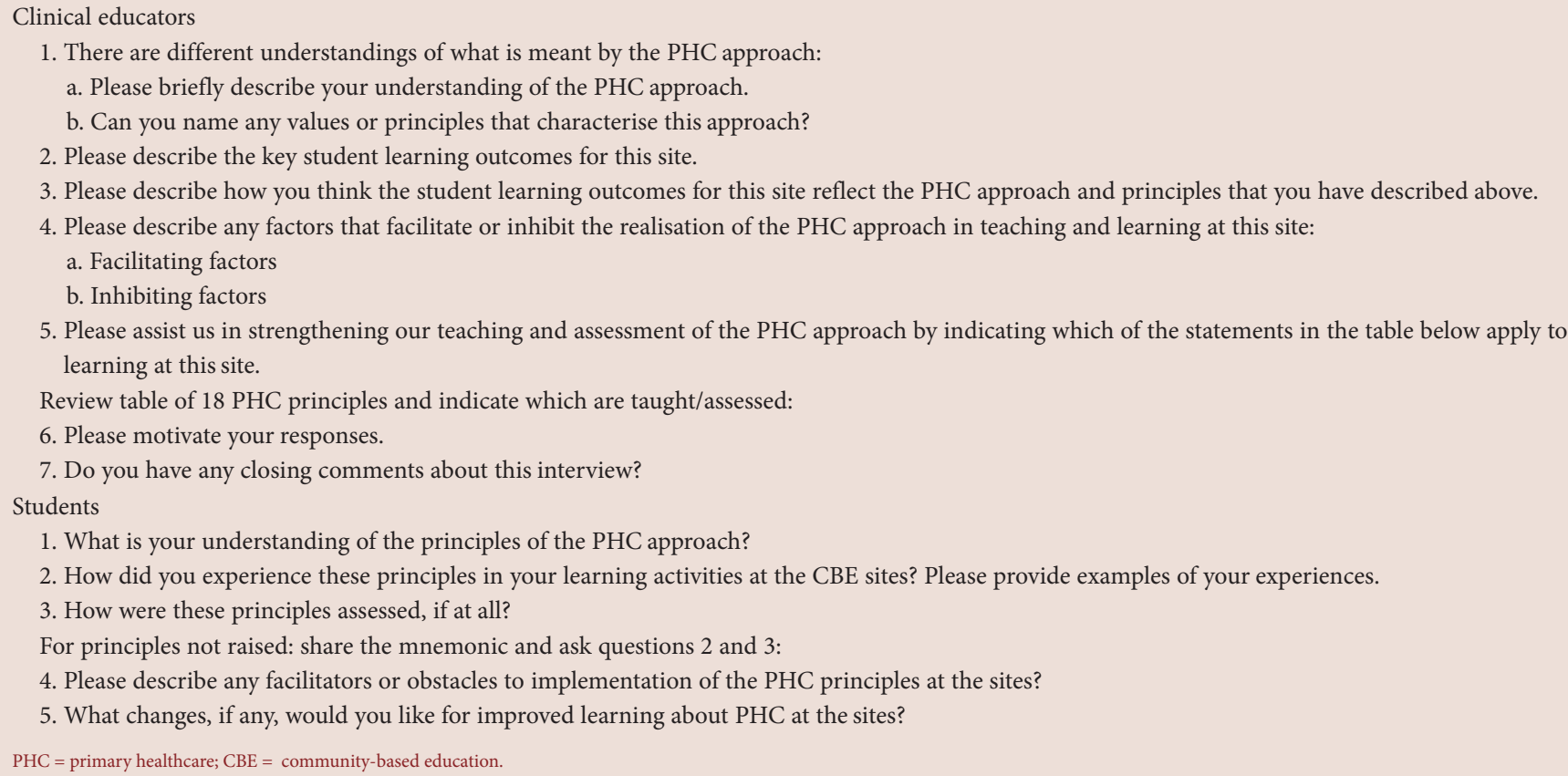

safely managing drips and catheters. PT course documents refer explicitly to the needs of communities, and CSD students are assessed on their community needs analyses. Although social accountability was not made explicit, CSD students undertake projects with community partners.

\section{Interviews with clinical educators}

\section{Educators' understanding of PHC approach}

Table 4 provides illustrative quotes from educator and student interviewees. For a clinical educator in O\&G at NSH, the PHC approach is evident in the multidisciplinary team that (s)he works alongside (quote 1). An experienced physiotherapy educator at VCHC referred to the team being able to recognise all the needs of the patient and to refer appropriately among themselves (quote 2). A qualified speech language therapist and audiologist who had convened CSD placements at VDH since 2013 emphasised partnerships with communities and non-governmental organisations, and the need for high-quality care that is evidence-based, cost-effective and respects the rights of patients and healthcare providers. The understanding of PHC as a holistic approach to care at all levels, rather than just primary-level care, is not always evident, however (quote 3). An educator at NSH observed that paediatrics registrars have no exposure to the PHC approach, and therefore do not explicitly teach it (quote 4).

\section{Educator perceptions of $\mathrm{PHC}$ in learning outcomes}

An experienced specialist physician at NSH who has taught medicine, physiology and pathology for a decade always teaches the multiple determinants of illness and healthcare behaviour, such as non-adherence (quote 5). An O\&G educator, who graduated from UCT over a decade previously, perceives more evidence of a holistic approach in the three preclinical years than during the clinical rotations at NSH during years $4-6$ (quote 6). She expects that students should broaden their approach by fifth year to include health promotion and prevention (quote 7).
An educator in SLT and audiology at VDH gave health promotion and disease prevention, especially in the form of counselling and educating mothers, as examples of PHC. A family physician at VDH believes that their multiprofessional (MP) team provides a holistic community-based service to all patients. Students also learn the importance of patient-centred care and making the most of available resources, such as learning to manage disease based on the essential drugs list. An OT educator at VDH spoke about the value of student exposure to community development with community-based OTs, specifically in early childhood development centres (quote 8).

A PT educator at VCHC believes that weekly observation of students' clinical skills and patient communication, followed by a feedback session, makes students more aware of the importance of respect, empathy and how to make therapy more effective. An experienced SLT educator gets her students to consider population health by doing group projects at various community-based sites (quote 9). She viewed the collaboration of PT, audiology, SLT and pharmacology students at the VCHC student learning centre as an important experience of MP teamwork.

\section{Educator perceptions of teaching and assessment of PHC principles}

Table 5 summarises educators' perceptions of the teaching and assessment of each of 18 PHC principles by site and discipline. The principles that are most often taught and assessed are: person-centred care; a multidisciplinary team approach; community participation; continuity of care; respecting human rights; appropriate care; evidence-based care; and promoting health by means of health education, behaviour change and public advocacy. Five principles that are most often taught but not assessed are: promoting equity and social justice; promoting broad intersectoral collaboration; affordable care; accessible care; and acceptable care. Principles that are seldom taught are: promoting health by means of policy reform; sustainably funded care; and environmentally sustainable care. 


\section{Research}

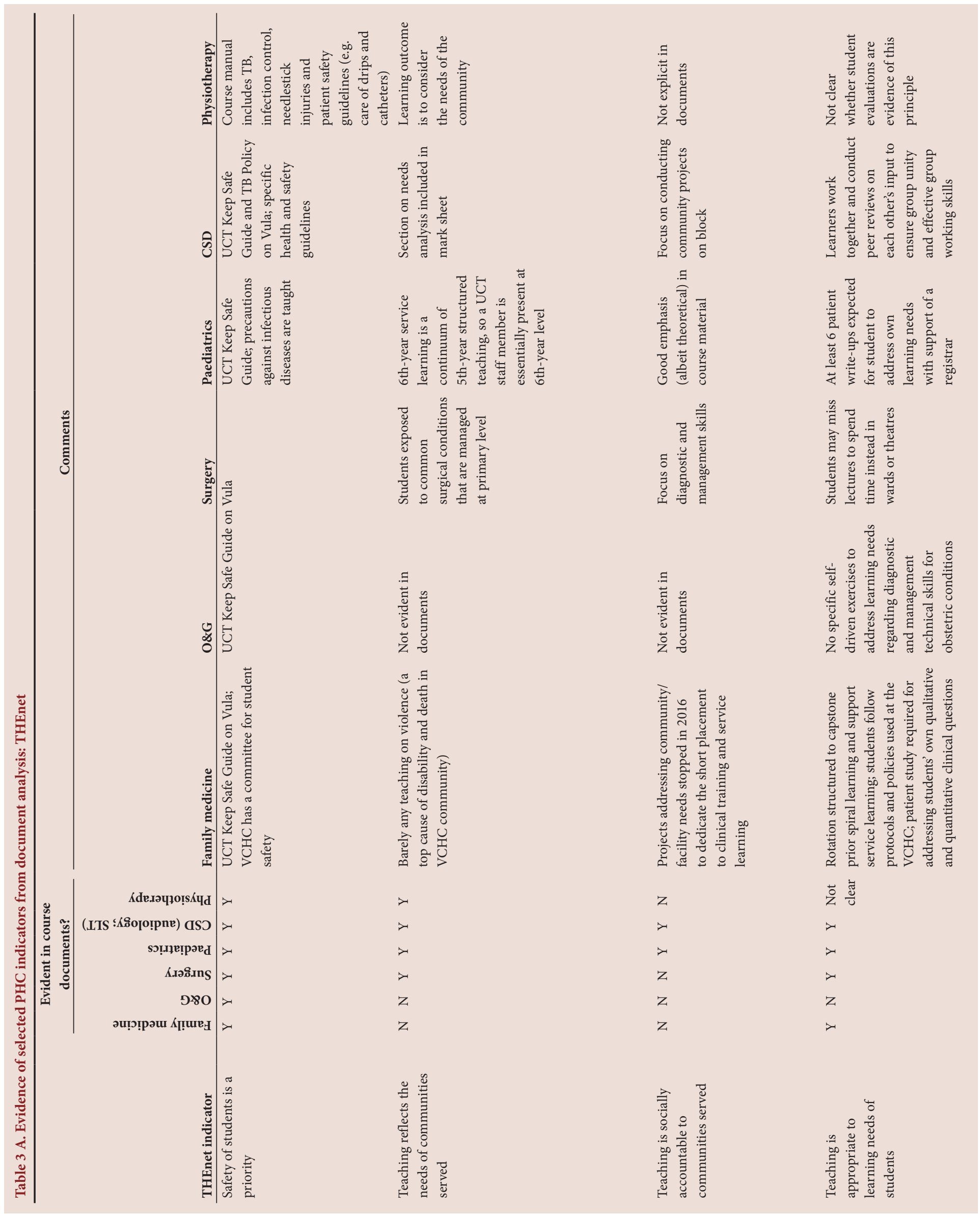




\section{Research}

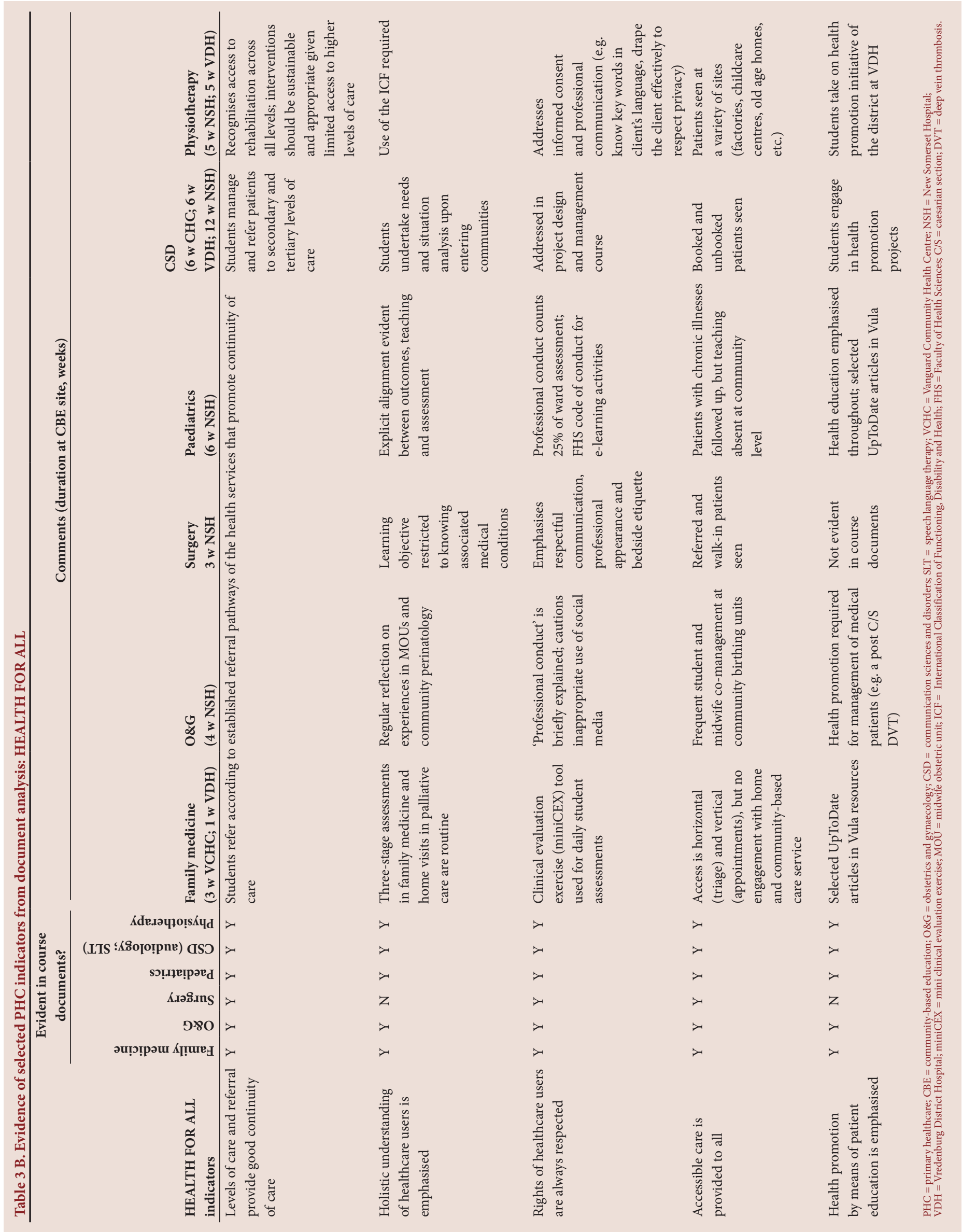


Table 4. Selected quotes from clinical educator and student interviews

\section{Clinical educators}

1. 'It cannot be done only by the medical doctor. We reach out to multiple areas in our discipline. The social workers are involved, and the physiotherapists are involved and dieticians are involved, and I think to me that is what primary healthcare represents.' (O\&G educator, NSH)

2. 'The patient should have availability or access to a combination of healthcare professionals to provide the basic services required; the interaction between those professionals should be able to recognise the needs of the patient and be able to refer amongst each other.' (PT educator, VCHC)

3. 'A lot of people get confused about the primary healthcare approach, confusing it with primary care and thinking this is something that only happens in clinics and at community level.' (speech language therapist, VCHC, NSH, VDH)

4. 'I was surprised to discover there is a primary health care directorate within UCT. You have a great website, and this is good stuff, but I have never heard of this and come across this before and seen the penetration of this through the undergraduate curriculum. The registrars don't know any of this and they get no exposure to it; and then when we teach as clinicians, we teach clinical work.' (paediatrics educator, NSH)

5. 'I always bring in the social and economic factors, religious, traditional factors that influence diseases and especially in our setting with TB and HIV. I remind the students of all the various factors that contribute to non-adherence to medications.' (general medicine educator, NSH)

6. 'I think we get this message (the importance of a biopsychosocial approach) across to the students actually quite strongly in the first 3 years. They take a little bit of time to get into it in the clinical years, but it is great if they get it all back at the end and it comes together. You get the students who don't focus on this at all, but I am sure there are consultants who do the same. And at the end of the day, I think we just need to reinforce that we cannot lose the primary healthcare principles in our everyday practice of medicine.' (O\&G educator, NSH)

7. 'I think if the student can take an adequate history and do a good examination, obviously problem-related, then they have achieved their goal in third and fourth year. In fifth year, it is much broader and I expect them to piece the puzzle together and make a diagnosis and come up with the management plan and then address all the key issues, including the health promotion and prevention issues.' (O\&G educator, NSH)

8. And within those centres in terms of the learning outcomes there is a lot related to the intention or philosophy of OT, but I think they mimic quite a few of the primary healthcare principles, in that it's about forming partnerships, building understanding as pivotal to forming a partnership, identifying occupational profiles of the children and their caregivers, which means gaining an understanding of not only what their skills are or their deficits, but how the context, the community, their home, their school, the broader community supports their health and wellbeing.' (OT educator, VDH)

9. 'When you are a hospital therapist it is actually a complete package in terms of seeing individual clients and doing group projects. These could be anything from providing information to breastfeeding mothers to explaining to them why it is beneficial to their babies, depending on what needs arise.' (speech language therapist, VCHC, NSH, VDH)

10. 'They learn how to make low-cost toys for example; they learn about assistive devices; they learn about different options that service users have to access different levels of healthcare. They know about the rights, say for example, of children to access health in the public sector for free beyond a certain age in line with their income.' (OT educator, VDH)

11. 'When I examine end-of-block and end-of-year exams and I do a portfolio exam and wrap up with what they learn from their PHC approach, they always say something quite glib and trite about intersectoral collaboration; or what this case taught me was this and that, and they say a very smug one-line sentence, which has probably been handed down and cut and pasted from years' worth of student portfolio cases, and we leave it at that. I don't teach explicitly PHC principles, and I don't explore their grasp and if they are able to come up with a principle and apply it to their case.' (paediatrics educator, NSH)

12. 'I think that is somewhere we can improve, somehow assess them more formally. I would think them seeing a patient and focusing then on primary healthcare would be a nice, practical way of them assessing their knowledge and understanding of primary healthcare'. (general medicine educator, NSH)

13. 'Clinical exposure is massively important, since students are listening to stories and listening to where people go when they are not in your office; and that helps them to actually understand how to adjust projects.' (speech language therapist, VCHC, NSH, VDH)

14. 'The intention in sending our final students there is because they gain a lot of the individual and population-based reasoning from them, and they've learnt already from community development practice about the value of gaining understanding, initiating, and working in a more developmental model. Sites such as Vredenburg give students the opportunity in a smaller kind of environment to work together, but also to live together and learn from each other.' (OT educator, VDH) Students

15. 'So for us we have devices that could help with speaking ... but they are not always affordable for all patients, so we need to think okay, what can we implement that will be suitable to the patient, that will be affordable.' (SLT student)

16. 'We all struggled with the language barrier ... for a lot of stroke patients who have aphasia, when you do the therapy that helps them, I don't know if you know about semantic feature analysis? The words just come out, and it was coming out in his home language, but now because he thought he had to do it in English, he kept shifting back to English and that's something that upset him, because now it's affecting his care and it's just, you don't realise how much language can affect, especially in the adult setting ... Then you might just diagnose them wrong, I mean it might come across as he has aphasia, which is not true, it's just that's not his first language. So you might just do something wrong. (SLT student)

17. 'So we in Vredenburg, Tuesday afternoons were set out for multidisciplinary team, interdisciplinary, the new word, that term that they use, interdisciplinary team meeting with the med students, the audiologists, physios and the speech therapists ... the first scenario that we had was a patient with TB and how you would treat them. And the doctors were like so what were the speech therapists and the audiologists doing here? And they were sitting there and they gave the facts, what medication they would give very briefly ... the speech therapist and audiologist came and we had this whole thing about feeding, if the patient is strong enough, if the patient is aspirating and then the audio said about the hearing that's going to get affected and if they are young and there's a long space of you not being able to hear, then that would affect the language. So the speech therapist and the audiologist took over that whole session, and we were just talking, and the doctors at the beginning were like but why are you here? And then after hearing all of these things, they were like oh, I didn't know you deal with feeding, or like oh? So it was such an eye opener for them and it was so nice for us to see that we could actually teach people about what we're doing, because there's such a small understanding of our job description, which is really nice to be able to advocate.' (SLT student)

18. 'We need more research and creating more culturally acceptable assessments, or translating assessments.' (SLT student)

$\mathrm{O} \& \mathrm{G}=$ obstetrics and gynaecology; NSH = New Somerset Hospital; PT = physiotherapy; VCHC = Vanguard Community Health Centre; VDH = Vredenburg District Hospital; UCT = University of Cape Town; $\mathrm{TB}=$ tuberculosis; $\mathrm{OT}=$ occupational therapy; $\mathrm{PHC}=$ primary healthcare; $\mathrm{SLT}=$ speech language therapy 
An OT educator at VDH believes that students are well assessed by means of written work and practical projects with respect to the affordability, accessibility, appropriateness, acceptability and environmental sustainability of care (quote 10; Table 4). Paediatrics students have to describe a PHC principle in their case report and portfolio examination, although an educator at NSH expressed some doubts about the validity of the assessment (quote 11). Another educator at NSH believes that formal assessment of the PHC principles should be improved (quote 12).

\section{Educator perceptions of factors that facilitate or inhibit PHC teaching and learning}

Most educators considered facilitating factors to be good MP teamwork, experienced health professionals who demonstrated the PHC approach, and the high burden of preventable disease that requires a preventive and health-promoting approach at all levels of care. The vulnerability of patients to diseases of poverty and underdevelopment, and to abuse by health professionals, was cited by some educators as motivating a strong focus on health equity and respect for patient rights. Other facilitating factors include well-run facilities, the purpose-built student learning centre at VCHC, wellestablished district outreach programmes, good monitoring of outcomes of care and reliable connectivity. Engaging with communities in their context is seen by an SLT educator to enhance understanding and the design of adaptive projects (quote 13, Table 4), a view shared by an OT educator in the rural VDH district, where collaborative learning in a more intimate and supportive environment adds great value to learning (quote 14).

The main barriers to PHC teaching and learning are lack of space in health facilities, excessive workload, understaffing, lack of critical staff, and inadequate and costly transport. Additional barriers were perceived to be a narrow biomedical focus in teaching, student safety in the community, poor referral pathways, inadequate student accommodation, insufficient outreach visits and suboptimal alignment between essential drug lists and tertiary treatment protocols.

\section{Interviews with final-year students}

All three students could define and share personal experiences of accessible, appropriate, affordable and holistic care. The SLT students also explained the multiprofessional and intersectoral principles of PHC. Immersion in the district at VDH helped students to appreciate patients in context, to better understand the referral system, and to see the importance of adapting patient management in low-resource and rural settings (quote 15, Table 4).

Role-modelling by experienced clinicians, as well as tutorials and formal discussions, enabled individual patient advocacy. While the two SLT students had advocated on behalf of school pupils and undertaken health promotion, this was not part of the medical student's experience, although he was aware of district health promotion activities. None had experience of broader advocacy such as public health campaigns. Continuity of care was evident in family medicine rotations at $\mathrm{VDH}$ and $\mathrm{VCHC}$, and by students referring to PHC principles in their written work. None, however, had specifically discussed PHC principles during ward rounds or in practice learning settings.

Language barriers constrain medical history-taking and SLT assessments, and hence the potential extent of patient or client-centredness (quote 16). Only the SLT students cited application of human rights principles. They described the weekly interdisciplinary team meetings at VDH at some length, 
where they experienced hierarchy, 'rank-pulling' and patronisation, and in practice settings as well (quote 17). They observed the consequences of lack of interdisciplinary referrals due to clinicians' insufficient understanding of the complementary roles of health professionals.

Constraints on practising evidence-based care were identified by the SLT students, who reported that several assessments needed to be adapted specifically for the SA context (quote 18). Although a few PHC principles are formally assessed in written work, all three students said that summative assessment of most of the principles was absent.

\section{Discussion}

\section{Document analysis}

PHC indicators are evident in the documented learning outcomes of finalyear medical students at the sites, but most principles are inconsistently taught within the disciplines reviewed. The 'spiral of learning' is particularly evident in $O \& G$ and paediatrics, progressing from mostly structured learning in earlier years to service-learning in the final year. Final-year teaching in PT and CSD is well scaffolded from previous years, and clinical expectations are built on an academic knowledge base. Use of the ICF may account for relatively good understanding in PT and CSD of the contextual factors that influence clinical assessment and management.

Services are made accessible: family medicine students do home visits for palliative care, PT students treat clients in a variety of workplace settings and SLT students see patients who have not been formally referred. The principle of social accountability is not evident, however, in the documents from three of the four medical disciplines. There were no documents that explicitly encourage students to engage with public health issues or with primary care strategies, such as the $90-90-90$ strategy $^{[11]}$ and the Healthcare 2030 strategy of the provincial Department of Health. ${ }^{[12]}$ Family medicine community projects, discontinued in 2016, cannot be reintroduced because the 4-week block is too short.

Graded PHC-related assessments are generally conducted by supervisors and sometimes by peers, but there are also non-graded assessment activities such as case discussions for formative feedback only. Neither the analysed documents nor the educator interviews provided sufficient clarity on which PHC principles are formatively assessed and which are assessed for marks.

\section{Interviews with clinical educators}

Educators' understanding of the PHC principles varied, yet most cited the importance of teamwork for holistic and accessible care at all levels, including health promotion and disease prevention. Continual awareness of the comprehensive PHC approach as a lead theme of the FHS is evidently needed among all clinical educators, however, so that the preclinical emphasis on the biopsychosocial approach, and opportunities for health education and promotion, are not lost during the clinical rotations.

Educators' perceptions of PHC principles in student learning outcomes, teaching and assessment reflected a good appreciation of their importance. Some excellent examples were shared of MP practice, community participation, respect for patient rights and health promotion, particularly at VDH and VCHC. There is, however, a missed opportunity for MP teamwork at VCHC with the absence of medical, OT and nursing students from weekly team meetings, which may be due to logistical difficulties arising from insufficient course planning across disciplines.
Nevertheless, the student learning centre at VCHC is generally considered to facilitate PHC teaching and learning, as is the supportive peer learning environment at VDH. Allowing students of different disciplines to interact and collaborate is evidently very beneficial for all teaching and learning, and should therefore be facilitated wherever possible.

\section{Interviews with final-year students}

The medical student appears to have had fewer opportunities to observe, experience and/or practise the PHC principles than the SLT students. The structure of the final-year curriculum allows medical students only 1 week of immersion at the VDH site, in contrast to 7 weeks for SLT students, with no opportunities for health promotion or home visits and only one MP session. Nevertheless, that 1 week of immersion was considered valuable for deepening understanding of holistic patient care. It was reported that some PHC principles were also taught and role-modelled at VCHC. All three students commented on limited formative assessment of the PHC principles, and their absence in summative assessment, which does not fully concur with the documents and perceptions of the educators. It may be that educators are not explicit enough about the assessments, or that students are not engaging sufficiently with the descriptors on the marksheets.

There is a fair degree of convergence overall between students' and educators' perceptions of facilitating factors for PHC. The SLT students' description of hierarchy in the weekly team meetings does not concur, however, with the opinion of most educators that role-modelling of good teamwork enables PHC teaching and learning. Furthermore, educators did not focus as much as students on language barriers to care. Other barriers are inadequate understanding of the comprehensive PHC approach, too few supervisors at some sites and insufficient time to engage in public health initiatives of the provincial Department of Health.

\section{Study limitations}

There are several limitations to this study. Firstly, only three CBE sites were studied, which although broadly representative of diversity across the clinical teaching platform, limits the generalisability of these findings. Secondly, the very low response rate among students, which we ascribe to deep trauma in the faculty following the former dean's recent untimely passing, meant that only one medicine and two SLT students with a predominant focus on one CBE site were interviewed. Thirdly, not all disciplines were represented in the samples of reviewed documents and educators interviewed.

Future research should therefore enlarge the sample of sites and disciplines, and strive particularly for many more student responses. It should also include the views of the communities it serves, which is essential if the FHS seeks to be more community-engaged. ${ }^{[13]}$

\section{Conclusion}

This study found that that final-year health sciences disciplines engage inconsistently with the selected PHC principles at the CBE sites. Alignment appears to be strongest between learning outcomes and teaching activities, particularly in the health and rehabilitation sciences, but there were insufficient data to judge whether there is also strong alignment with formal graded assessment. This finding needs further research and attention in future curriculum planning, as any misalignment introduces a 'hidden curriculum', which may signal that the PHC principles are of lesser importance than other learning outcomes. ${ }^{[14]}$ 
The eight educators and three students who were interviewed perceived that PHC teaching and learning is facilitated by good multiprofessional teamwork, educator role-modelling and good infrastructural and logistical support. Language barriers and health system issues such as staff shortages and high workloads are prevalent barriers to PHC teaching and learning on the CBE platform.

We recommend strong faculty leadership to promote the PHC lead theme and to achieve better departmental and multiprofessional collaboration in teaching the PHC approach. A new dean and two deputy deans in the FHS provide a good opportunity for the PHC directorate to strengthen the PHC approach under new leadership, with a strong grounding in social accountability. This work should be done in alignment with the FHS strategic plan, as two of its five goals are directly relevant to PHC: to develop the decentralised teaching and learning platform for $\mathrm{CBE}$, including a rural campus; and to promote the PHC approach in teaching, research, health service partnerships and community engagement. Recommendations of the 2018 faculty-wide symposium that are endorsed by the action research group include: providing training and support for all educators in the PHC approach; ensuring that PHC is explicit in all curriculum documents; integrating social accountability into the key performance areas of academic staff, including heads of department; and regular (5-yearly) reviews of FHS governance with respect to the PHC principles. The Academy of Science of SA (ASSAf) report of 2018 (Reconceptualising Health Professions Education in SA) also provides practical recommendations on addressing the inequitable national shortages of healthcare professionals, which include prioritising applicants from rural and underserved areas, and strengthening education for practice in such areas. ${ }^{[15]}$

The present study provides important and timely evidence from wellestablished CBE sites in the FHS to inform future work and participatory action research in promoting the PHC approach in teaching and learning in the FHS.

Declaration. None.

Acknowledgements. Fatima le Roux, NSH site co-ordinator; all clinical educator and student participants.
Author contributions. All authors complied with the International Committee of Medical Journal Editors' rules of authorship and were part of formulating and conceptualising the article. The initial draft was prepared by the first author, and subsequent work on the manuscript included inputs from all authors.

Funding. None.

Conflicts of interest. None.

1. World Health Organization. Declaration of Alma-Ata: International Conference on Primary Health Care Alma-Ata, USSR, 6 - 12 September 1978. https://www.who.int/publications/almaata_declaration_en.pd (accessed 23 February 2021)

2. Irlam J, Keikelame MJ, Vivian L. Integrating the primary health care approach into a medical curriculum: A programme logic model. Afr J Health Professions Educ 2009;1(1):8-11.

3. Frenk J, Chen L, Bhutta ZA, et al. Health professionals for a new century: Transforming education to strengthen health systems in an interdependent world. Lancet 2010;376(9756):1923-1958. https://doi.org/10.1016/S01406736(10)61854-5

4. Ritz SA, Beatty K, Ellaway RH. Accounting for social accountability: Developing critiques of socia accountability within medical education. Educ Health 2014;27(2):152-157. https://doi.org/10.4103/13576283.143747

5. Boelen C, Woollard B. Social accountability and accreditation: A new frontier for educational institutions. Med Educ 2009;43(9):887-894. https://doi.org/10.1111/j.1365-2923.2009.03413.x

6. Boelen C. Why should social accountability be a benchmark for excellence in medical education? Educación Médica 2016;17(3):101-105. https://doi.org/10.1016/j.edumed.2016.06.004

7. Training for Health Equity Network (THEnet). Framework for Socially Accountable Health Workforce Education, ver. 2. THEnet, 2016. https://thenetcommunity.org/the-framework/ (accessed 23 Februar

8. Yin R. Case Study Research: Design and Methods. Thousand Oaks, CA: Sage Publications Inc., 2003.

9. Baxter P, Jack S. Qualitative case study methodology: Study design and implementation for novice researchers. Qual Rep 2008;13(4):544-559.

10. World Health Organization. International classification of functioning, disability and health: ICF. Geneva WHO, 2001.

11. Ajayi A, Mudefi E, Adeniyi O, et al. Achieving the first of the Joint United Nations Programme on HIV/AIDS (UNAIDS) 90-90-90 targets: Understanding the influence of HIV risk perceptions, knowing one's partner's status and discussion of HIV/sexually transmitted infections with a sexual partner on uptake of HIV testing. Int Health 2019;11(6);425-431. https://doi.org/10.1093/inthealth/ihz056

12. Western Cape Department of Health. Healthcare 2030: The road to wellness. Cape Town: WCDoH, 2014. https://www.westerncape.gov.za/assets/departments/health/healthcare2030_0.pdf (accessed 23 February 2014.

13. Strasser R, Worley, P, Cristobal F, et al. Putting communities in the driver's seat: The realities of community-engaged medical education. Acad Med 2015;90(11):1466-1470. https://doi.org/10.1097 0000076

14. Alsubaie MA. Hidden curriculum as one of current issue of curriculum. J Educ Pract 2015;6(33):125-128

15. Academy of Science of South Africa. Reconceptualising health professions education in South Africa. Pretoria: ASSAf, 2018. http://research.assaf.org.za/handle/20.500.11911/95 (accessed 23 February 2021). 